\title{
UTILIZAÇÃO DE SENSOR DE FALTA PARA LOCALIZAÇÃO DE DEFEITOS EM REDES DE MÉDIA TENSÃO
}

\section{USE OF FAULT SENSOR TO LOCATE DEFECTS IN MEDIUM VOLTAGE NETWORKS}

\author{
Álvaro Pereira Milani ${ }^{1}$, Bruno Flores Farinazzo ${ }^{2}$, Fábio Batista de Oliveira ${ }^{3}$, Mateus de Sousa \\ Cardoso ${ }^{4}$, Antônio Elízio de Oliveira ${ }^{5}$
}

\footnotetext{
${ }^{1}$ Graduando em Engenharia Elétrica, Universidade do Sul de Minas, Cataguases, MG, Brasil, alvaromilani93@gmail.com*

${ }^{2}$ Graduando em Engenharia Elétrica, Universidade do Sul de Minas, Cataguases, MG, Brasil, brunoflored@icloud.com

${ }^{3}$ Graduando em Engenharia Elétrica, Universidade do Sul de Minas, Cataguases, MG, Brasil, oliveirabf@yahoo.com.br

${ }^{4}$ Graduando em Engenharia Elétrica, Universidade do Sul de Minas, Cataguases, MG, Brasil, mateuscardoso@energisa.com.br

${ }^{5}$ Coordenador de Pesquisa, Universidade do Sul de Minas, Cataguases, MG, Brasil, antonio.oliveira@professor.unis.edu.br
}

\begin{abstract}
Resumo
Este artigo tem por objetivo analisar a aplicabilidade de sensores de falta em redes de distribuição de média tensão em uma concessionária de energia elétrica na zona da mata mineira. Foram utilizados para o estudo os dados hipotéticos para análise de redução de DEC da ferramenta, bem como o acervo digital relacionado ao tema, fato pelo qual esclarece tecnicamente as características do equipamento. A aplicação do sensor supre uma necessidade evidente nas distribuidoras de energia elétrica, uma vez que identificar falhas em sistemas elétricos de distribuição - principalmente à noite, é um desafio real e iminente a todas.
\end{abstract}

Palavras-chave: Sensor de falta. Média Tensão. Sistema Elétrico.

\begin{abstract}
This article aims to analyze the applicability of fault sensors in medium voltage distribution networks in an electric power utility in the region of the forest of Minas Gerais. The hypothetical data for the analysis of the tool's DEC reduction were used for the study, as well as the digital collection related to the topic, a fact that technically clarifies the characteristics of the equipment. The application of the sensor fulfills an evident need in electrical energy distributors, since identifying faults in electrical distribution systems - especially at night, is a real and imminent challenge for all.
\end{abstract}

Keywords: Fault sensor. Medium Voltage. Electrical system.

CUNIS-MG. All rights reserved.

How to cite this article:

MILANI, Álvaro Pereira. FARINAZZO, Bruno Flores. OLIVEIRA, Fábio Batista. CARDOSO, Mateus de Sousa. DE OLIVEIRA, Antônio Elizio. Utilização de sensor de falta para localização de defeitos em redes de média tensão. Revista Mythos, Cataguases, MG, v. 10, p. xxx, 2019. ISSN 1984-0098

Disponível em: https:// endereço do periódico. Acesso em xx de xxx de 20XX.

DOI: https:// colocar o doi do artigo (editoração da revista) 


\section{INTRODUÇÃO}

O sistema elétrico brasileiro, desde sua geração de energia elétrica à sua distribuição em si, enfrenta desafios de pequena, média e elevada dificuldade. Desta forma, há de caracterizar que todo estudo relacionado ao tema é de grande valia para todo o segmento. A Agência Nacional de Energia Elétrica - ANEEL, autarquia vinculada ao Ministério de Minas e Energia, é o regulador do setor elétrico brasileiro e dispõe de critérios específicos para apurar, entre outras coisas, a continuidade do serviço distribuição de energia elétrica no Brasil.

A ANEEL iniciou suas atividades em dezembro de 1997, tendo como principais atribuições:

- Regular a geração (produção), transmissão, distribuição e comercialização de energia elétrica;

- Fiscalizar, diretamente ou mediante convênios com órgãos estaduais, as concessões, as permissões e os serviços de energia elétrica;

- Implementar as políticas e diretrizes do governo federal relativas à exploração da energia elétrica e ao aproveitamento dos potenciais hidráulicos;

- Estabelecer tarifas;

- Dirimir as divergências, na esfera administrativa, entre os agentes e entre esses agentes e os consumidores, e

- Promover as atividades de outorgas de concessão, permissão e autorização de empreendimentos e serviços de energia elétrica, por delegação do Governo Federal.

Considerando toda a abrangência e necessidades de se manter a qualidade e continuidade da energia elétrica aos consumidores, o estudo de caso em questão está relacionado a utilização de sensores de falta em rede de média tensão na rede de uma concessionária de energia elétrica que atua no interior de Minas Gerais, e tem como prioridade destacar os principais objetivos do equipamento, bem como relacionar os dados quantitativos relacionado aos dados históricos e sua percepção futura. Assim, este sistema deverá ser capaz de monitorar as faltas em linhas de distribuição, seja do tipo permanente quanto temporária, além de indicativo de tensão na linha, medição de corrente e sinalização de sobrecorrente de fase e desequilíbrio, sentido de carga e desequilíbrios que podem ser originários de faltas de alta impedância.

\section{REVISÃO DE LITERATURA}

\subsection{Indicadores de Continuidade}

Conforme disposto no PRODIST - Módulo 8, seção 8.2, capítulo 5, subitem 5.8.5, é realizada anualmente a apuração dos Desempenho Global de Continuidade - DGC pela Agência Nacional de Energia Elétrica - ANEEL, que é o órgão regulador do setor de Distribuição de Energia Elétrica brasileiro, e publicado até abril de cada ano. Este índice é obtido através da razão dos valores realizados de DEC e FEC por seus respectivos limites, e após sua apuração compõe o ranking da continuidade onde as empresas são classificadas, de acordo com seu porte (quantidade de clientes) e o índice realizado (quanto menor o índice DGC melhor a empresa se mostrou no período de apuração e será mais bem classificada). Conforme a ANEEL (2016), “(...), o DGC visa comparar o desempenho de uma distribuidora em relação às demais empresas do país. $O$ indicador permite avaliar o nível da continuidade da distribuidora (...)".

Para as Distribuidoras é muito importante estar mais bem colocado no ranking da continuidade pois este fato vai além simplesmente do índice apurado, mas também está relacionado a possibilidade 
de ganhos financeiros pelo melhor desempenho período de apuração, através do Fator $X$ e de seu Componente Q. De acordo com a ANEEL (2015), “O Fator X tem como objetivo primordial a garantia de que o equilíbrio estabelecido na revisão tarifária entre receitas e despesas eficientes seja mantido nos reposicionamentos tarifários subsequentes". Já o componente $Q$, visa ser um mecanismo de incentivo a melhoria da qualidade do serviço prestado pelas distribuidoras, conforme ANEEL (2015) através do PRORET - Procedimentos de Regulação Tarifária, Módulo 2, Submódulos 2.5 e $2.5 \mathrm{~A}$, de acordo com o ano da revisão tarifária da Distribuidora.

Diante deste cenário cada vez mais competitivo e de comparação, as empresas Distribuidoras de energia buscam rotineiramente desenvolver soluções que reduzam o impacto de eventos externos e que geram interrupções a seus clientes e, consequentemente, trazendo impactos aos seus indicadores de continuidade, de forma a garantir o cumprimento dos índices de qualidade do fornecimento (DEC e FEC), impostos pelo órgão regulador.

O PRODIST Módulo 8 é subdividido em duas seções. 0 artigo em questão está relacionado a seção 2, referente a Qualidade do Serviço prestado pelas Distribuidoras em termos de duração (DIC-DMICDEC) e frequência (FIC-FEC). Conforme o PRODIST, Módulo 8, Seção 8.2 (2018), temos:

○ DIC - Duração de Interrupção Individual por Unidade Consumidora ou por Ponto de Conexão, expressa em horas e centésimos de hora;

○ FIC - Frequência de Interrupção Individual por Unidade Consumidora ou por Ponto de Conexão, expressa em número de interrupções e centésimos do número de interrupções;

○ DMIC - Duração máxima de Interrupção Contínua por Unidade Consumidora ou por Ponto de Conexão expressa em horas e centésimos de hora;

- DEC - Duração Equivalente de Interrupção por Unidade Consumidora, expressa em horas e centésimos de hora;

○ FEC - Frequência Equivalente de interrupção por Unidade Consumidora, expressa em número de interrupções e centésimos do número de interrupções.

De acordo com o exposto acima, a apuração dos indicadores de continuidade é realizada de forma individualizada (DIC e FIC) e, através destes indicadores individuais é realizada a consolidação dos indicadores coletivos (DEC e FEC), que medem a duração e a frequência média que cada unidade consumidora da Distribuidora teve seu fornecimento de energia elétrica interrompido no período de apuração. Na Figura 1 é possível conhecer as fórmulas de cálculo do DEC e FEC, conforme PRODIST Módulo 8, seção 8.2, item 5.5 (2018): 


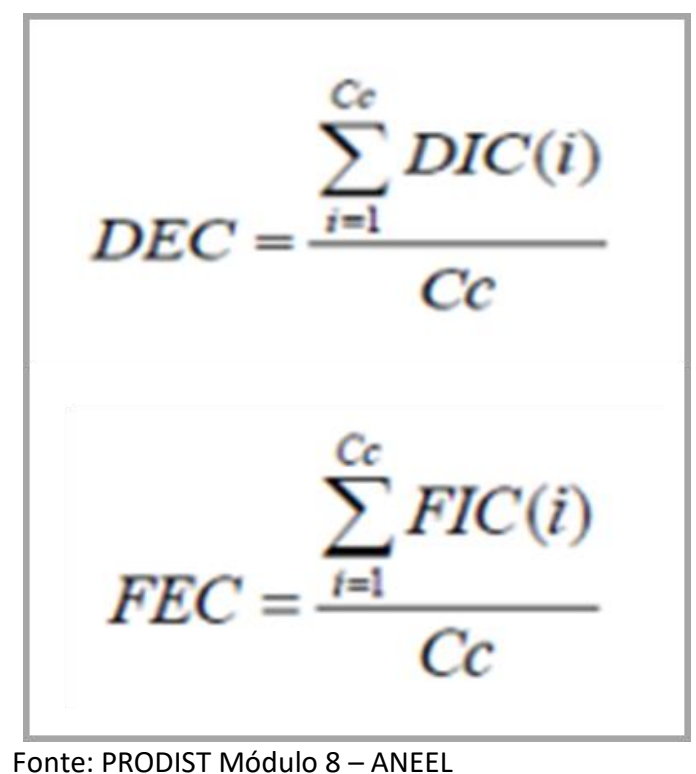

Figura 1 - Fórmula DEC e FEC

Onde:

i = índice de unidade consumidoras atendidas em BT ou MT faturadas do conjunto;

$\mathrm{Cc}=$ número total de unidades consumidoras faturadas do conjunto no período de apuração, atendidas em BT ou MT.

Para o correto entendimento e interpretação do cálculo dos indicadores de continuidade coletivos é necessária a compreensão do conceito de agrupamento dos consumidores de cada área de concessão. Conforme PRODIST - Módulo 1 (2008), temos a descrição de conjunto de unidades consumidoras como "Agrupamento de unidades consumidoras, aprovado pela ANEEL e pertencente a uma mesma área de concessão ou permissão".

Atualmente, a regra de formação de conjuntos de unidades consumidoras ou conjuntos elétricos tem relação com as Subestações de Distribuição (SED) e os critérios para formação dos mesmos, conforma PRODIST Módulo 8, são:

i. Abrangência do conjunto deve ser as redes média tensão - MT à jusante da SED e de propriedade da distribuidora.

ii. SED que possuam números de unidades consumidoras igual ou inferior a 1.000 clientes devem ser agrupadas a outras, formando um único conjunto.

iii. SED com número de unidades consumidoras superior a 1.000 e igual ou superior a 10.000 podem ser agregadas a outras, formando um único conjunto.

iv. A agregação de SED deve obedecer ao critério de contiguidade das áreas.

v. É vedada a agregação de duas ou mais SED cujos números de unidades consumidoras sejam superiores a 10.000

vi. Mediante aprovação da ANEEL, poderão formar diferentes conjuntos SED que atendam a áreas não contíguas, ou que atendam a subestações MT/MT. 
Vale lembrar que para cada conjunto elétrico de uma Distribuidora de Energia Elétrica existem limites regulatórios para os indicadores de continuidade (DEC e FEC), ou seja, indicadores máximos que em média as unidades consumidoras de determinados conjuntos podem ter seu fornecimento interrompido e por um tempo máximo determinado.

Conforme ANEEL (2015), a definição de limites é a criação de "Indicadores definidos pela ANEEL para aferir a qualidade do fornecimento de energia elétrica no conjunto de unidades consumidoras da área de atuação de cada distribuidora. Consideram a duração e frequência das interrupções no fornecimento do serviço". Estes limites são definidos através da realização de audiências públicas em fase de revisões tarifárias, por concessionária, que ocorrem de 4 em 4 anos ou de 5 em 5 anos, a depender do contrato de concessão de cada distribuidora. Após as revisões tarifárias, são publicadas resoluções autorizativas com os limites de DEC e FEC para cada conjunto.

\subsection{Princípio de funcionamento dos Sensores de Falta}

Segundo Bertulucci (2016), sensores são dispositivos capazes de detectar, com eficiência, entradas provenientes de ambientes físicos, com capacidade de converter sinais que podem ser lidos por processadores, eletronicamente, emitir sinais sonoros ou até mesmos luminosos. Este equipamento, por sua vez, proporciona maior segurança operacional ao sistema e reduz o tempo de interrupções no fornecimento de energia.

Conforme IEEE, entende-se como "detecção de falta" o reconhecimento do sensor de uma interrupção de fornecimento de energia elétrica ocasionado pela atuação de um de seus equipamentos de proteção, como por exemplo, abertura de uma chave seccionadora devido a um curto-circuito. Desta forma, o sensor de falta identificará o local exato que provocou a atuação do equipamento, bem como sinalizar se foi a jusante ou a montante da sua posição no alimentador. Ao identificar a falta, o sensor acende uma luz em LED, sinalizando aos eletricistas da concessionária o local da falha.

Conforme podemos observar na figura 2, a estrutura operativa dos sensores/indicadores de falta identifica se houver excesso de sobrecorrente acima do trip ajustado no indicador de falta, fazendo com que o equipamento seja ativado e sinalize a falta.

Na prática, considerando que a Distribuidora local dispõe de equipes técnicas específicas para reestabelecimento em casos de ocorrência, ela procura e localiza a falta isolando a seção da linha entre o último indicador de falta ativado (em vermelho) e o primeiro indicador de falta não ativado (em branco). 


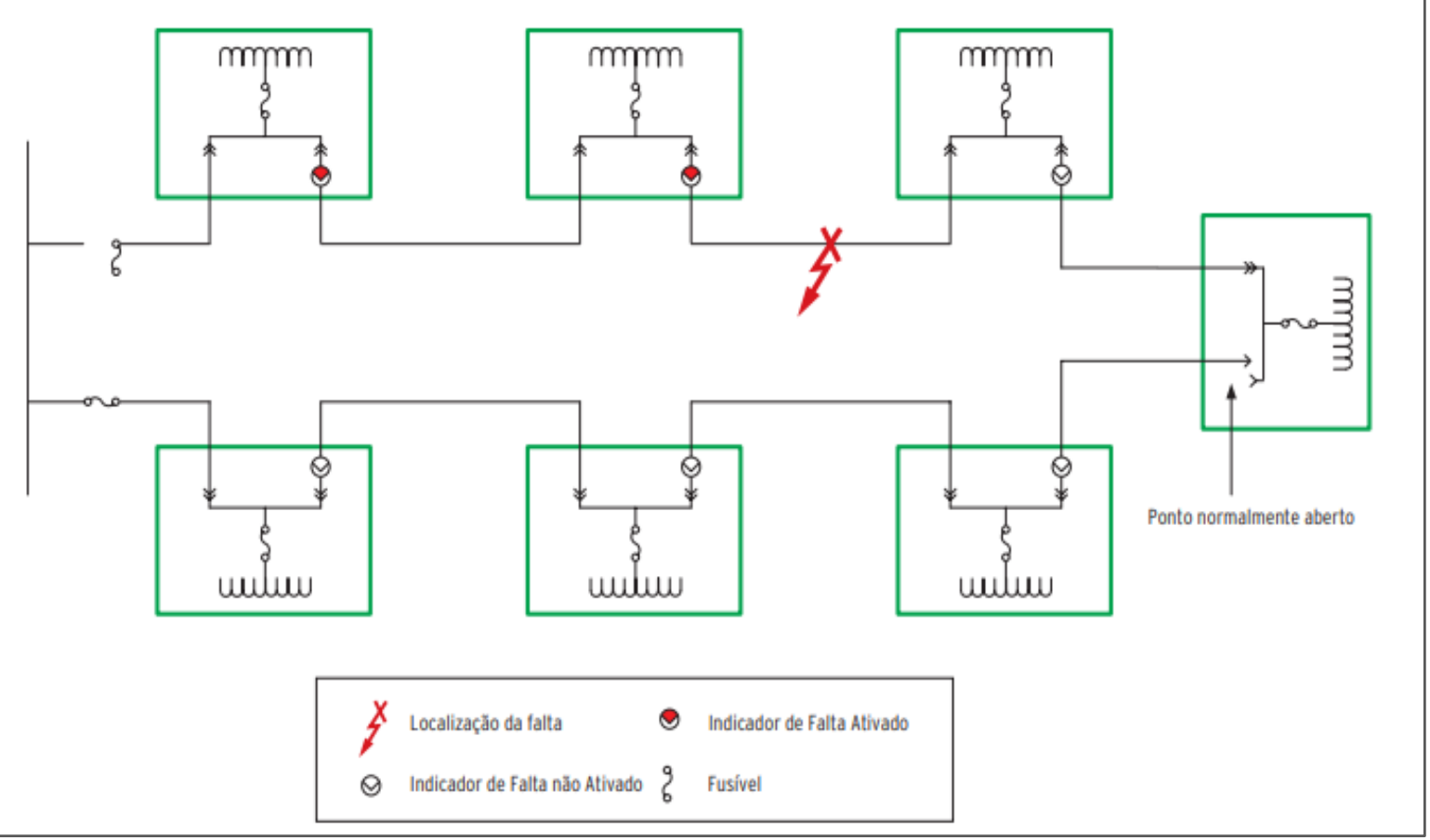

Fonte: SCHWEITZER MANUFACTURING, Sensores e Indicadores de Falta. Catálogo 2008.

Figura 2 - Aplicação dos sensores de falta

\section{METODOLOGIA}

A pesquisa a qual se refere este estudo está pautada no acervo disponível em meios digitais, catálogos de fornecedores de materiais elétricos e conteúdo dos órgãos reguladores (Agência Nacional de Energia Elétrica - ANEEL e Ministério de Minas e Energia - MME), dados técnicos de registros de ocorrência de Distribuidora de Energia Elétrica do interior de Minas Gerais, buscando identificar as ocorrências significativas e o impacto aos consumidores. A proposta em se aplicar sensores de falta em linhas de distribuição de energia elétrica justifica-se pela necessidade de se reestabelecer de forma breve o fornecimento de energia, seja pelos parâmetros regulatórios que penalizam as distribuidoras por descumprimento de indicadores de continuidade (ressarcimento financeiro aos consumidores por meio das violações do limite de seus indicadores individuais), pelo objetivo de garantir a qualidade no fornecimento aos seus clientes.

\section{SOLUÇÃO APLICADA}

Como citado, os sensores de falta possuem indicadores que facilitam a visualização pela equipe operacional de uma Distribuidora, durante o atendimento de uma ocorrência de falta de energia em determinada região.

A figura 3 ilustra o sensor de falta de modelo Autoranger 360 do fabricante Schweitzer Engineering Laboratories (SEL COMERCIAL). Este sensor é aplicado em redes aéreas de distribuição e simplifica a identificação de faltas temporárias: o LED amarelo pisca quando ocorre uma falta temporária. 


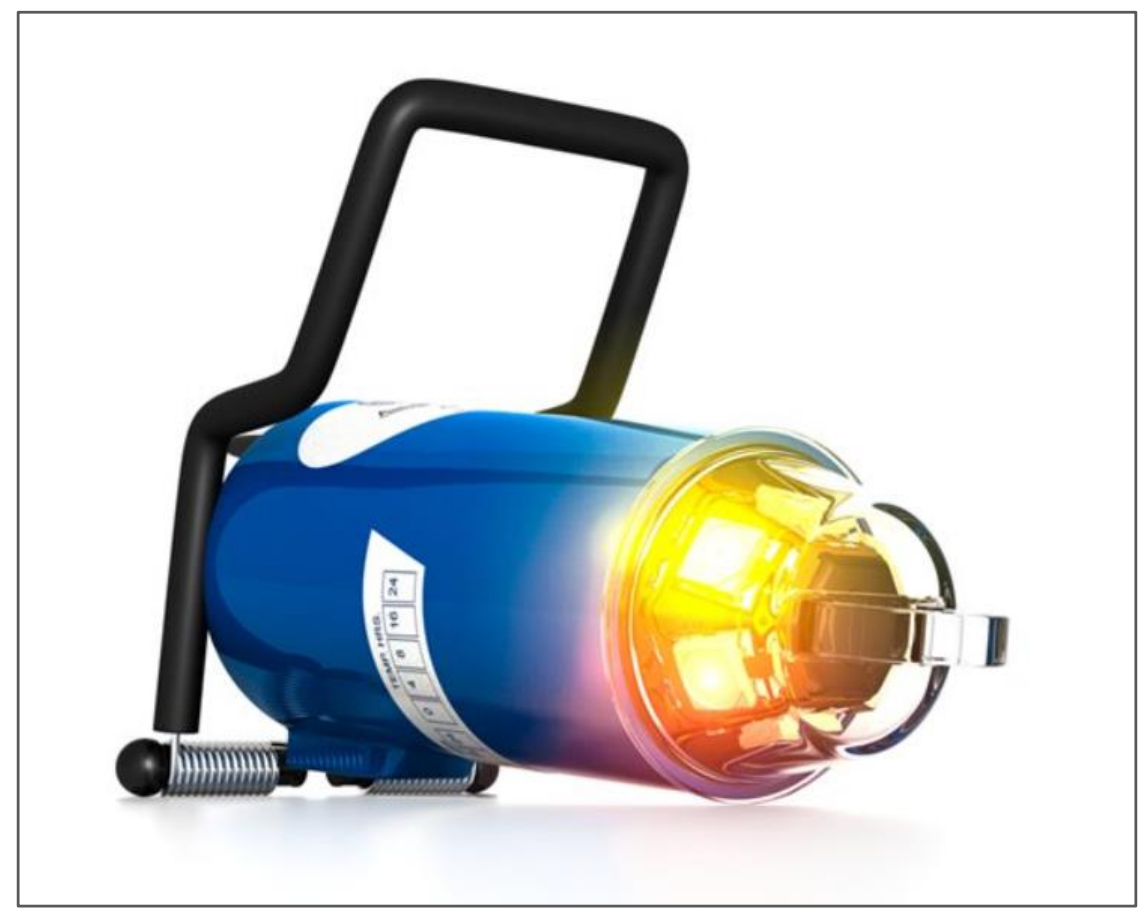

Fonte: Portal do Fabricante

Figura 3 - Sensor de falta utilizado para estudo

Os principais benefícios destes sensores são:

- Níveis de trip autoajustáveis baseados na corrente de carga medida;

- Indicação distinta de falta permanente e temporária;

- Alta visibilidade, display com LED de intensidade autoajustável;

- Ativação imediata na instalação;

- Instalação simples e sem manutenção;

- Entre outros.

As faltas temporárias e permanentes são identificadas pelos sensores separadamente através do uso de LEDs nas cores vermelho e âmbar, com padrões de flashes diferenciados. As faltas permanentes são indicadas por uma combinação de LEDs vermelho e âmbar alternados. Através destas indicações, é possível detectar as faltas momentâneas antes que se tornem interrupções permanentes, agilizando a normalização do sistema.

Conforme figura 4, abaixo, os sensores de falta são instalados na rede de média tensão, sendo inserida uma peça em cada fase com o auxílio de uma vara de manobra isolada, não sendo necessário acessórios e equipamentos extras para fixação. 


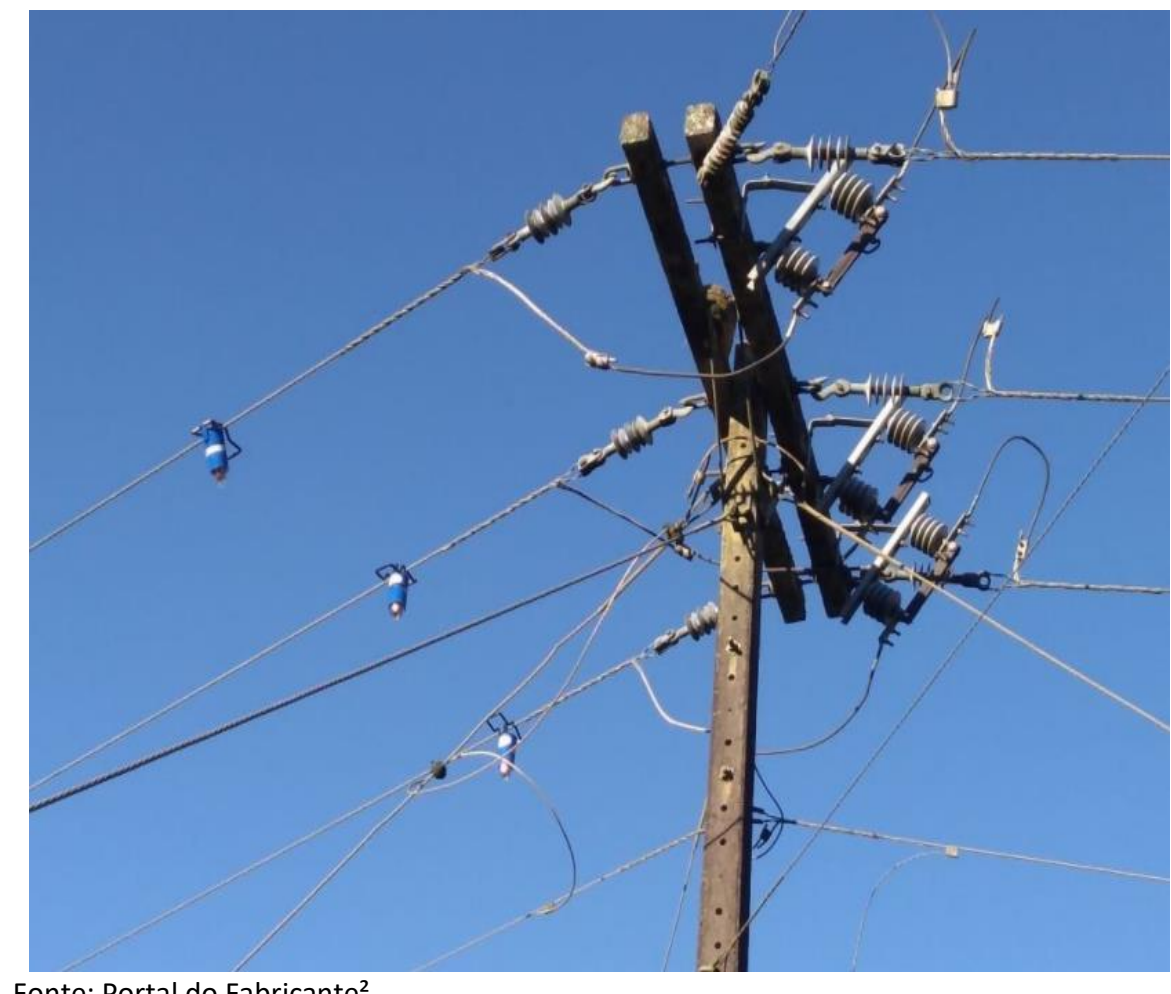

Fonte: Portal do Fabricante ${ }^{2}$

Figura 4 - Sensores instalados na rede de média tensão

Em alguns modelos de sensores de falta a visualização da falha é ainda melhor devido esses equipamentos possuírem em seu funcionamento um recurso de sequência de flashes "rotativos", permitindo a visibilidade do sensor em 360․

Considerando o constante desafio das Distribuidoras em manterem excelentes patamares de qualidade do fornecimento de energia elétrica, faz-se necessário o investimento em tecnologias que contribuam para o restabelecimento da energia elétrica o mais rápido possível.

Dessa forma, foi realizada uma análise dos potenciais ganhos com base na utilização do sensor de falta em determinado alimentador de uma Distribuidora de energia elétrica da Zona da Mata Mineira.

\section{RESULTADOS OBTIDOS E DISCUSSÃO}

Conforme citado nos desenvolvimento deste artigo, os sensores de falta são utilizados, em sua maioria, em circuitos alimentadores tipicamente rurais onde as distâncias são grandes entre as chaves de manobras, cujo existem dificuldades de deslocamento devido a fatores intrínsecos e na localização dos defeitos, além da quantidade de interrupções ser maior e mais frequentes devido a incidência de grande volume de área arborizada, impactando nos indicadores de continuidades das Distribuidoras.

2 Disponível em: < https://selinc.com/pt/company/news/133911/ .. Acesso em 26 de outubro de 2021.

A seguir, na figura 5, destaca-se um diagrama unifilar com um exemplo didático hipotético, exemplificando o ganho da utilização deste equipamento em um circuito alimentador tipicamente 
rural de uma concessionária de energia elétrica do interior de Minas Gerais. Foi simulado dois circuitos, denominados "Circ. 1" e "Circ.2" conectados ao barramento de uma subestação, no qual existem diversos blocos que representam os alimentadores de distribuição, estando a eles conectados diversos consumidores. $\mathrm{O}$ defeito, para este exemplo é aplicado após o sinalizador de falta, entre as chaves 004 e 005 e está afetando diretamente o bloco 1D.

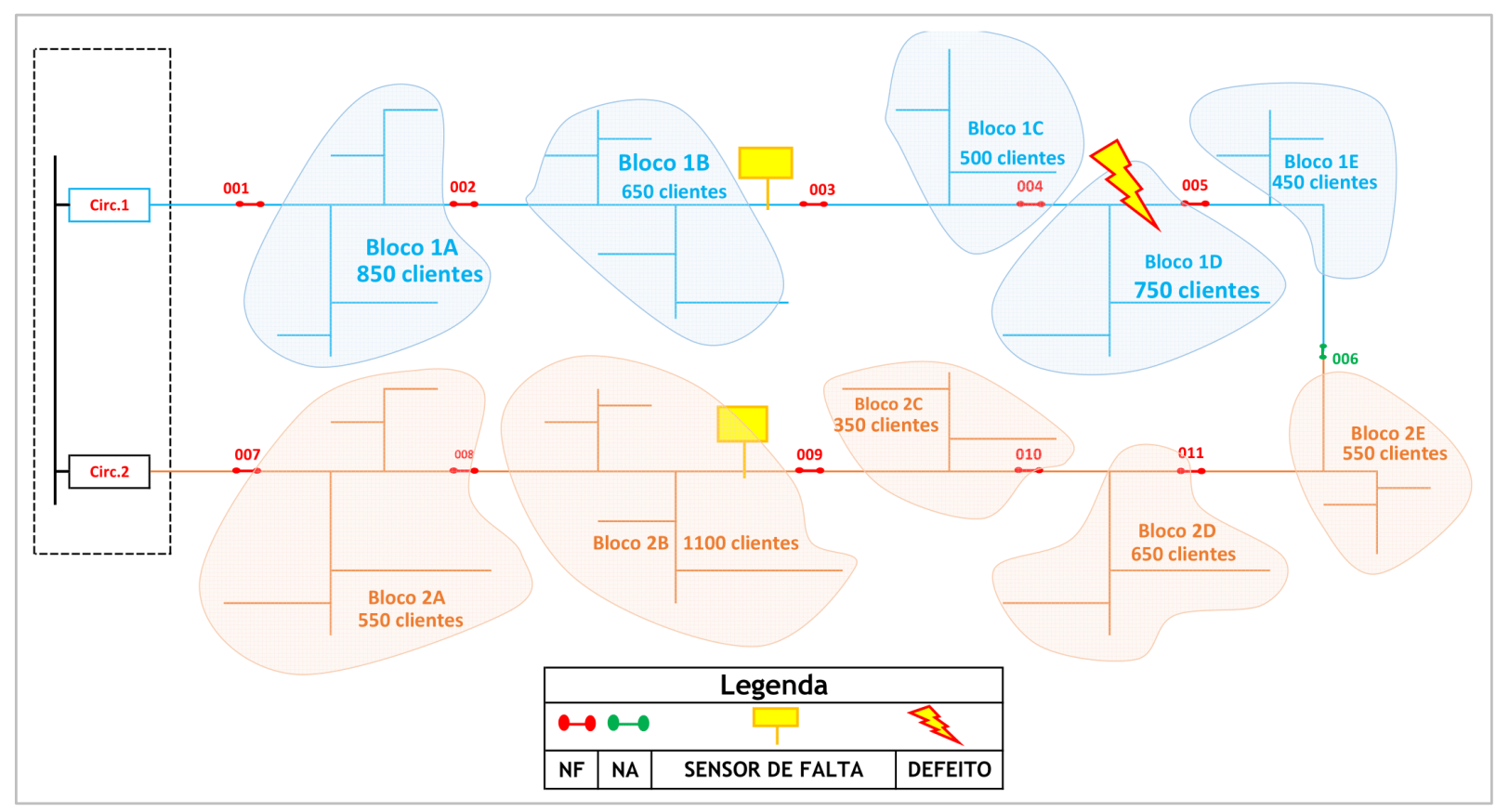

Fonte: Próprios Autores

Figura 5 - Diagrama unifilar - simulação de defeito entre chaves 004 e 005 no circuito 1

A seguir, na figura 6, estão dispostas as informações gerais sobre a quantidade de clientes (blocos) e distância entre as chaves, assim com o tempo de deslocamento/inspeção entre as chaves. Estas informações são importantes para simulação dos tempos entre as manobras de recomposição do sistema, para que assim seja possível calcular o impacto das interrupções nos indicadores de desempenho da Distribuidora.

\begin{tabular}{|c|c|c|c|c|c|}
\hline Circuito & Bloco & Qte Clientes & Distância (km) & Trecho & Tempo Inspeção (min) \\
\hline \multirow{5}{*}{1} & A & 850 & 10 & Entre chave 001 e 002 & 30 \\
\hline & B & 650 & 12,5 & Entre chave 002 e 003 & 38 \\
\hline & C & 500 & 7,5 & Entre chave 003 e 004 & 23 \\
\hline & D & 750 & 5 & Entre chave 004 e 005 & 15 \\
\hline & $\mathrm{E}$ & 450 & 5 & Entre chave 005 e 006 & 15 \\
\hline \multirow{5}{*}{2} & A & 550 & 8,5 & Entre chave 007 e 008 & 25,5 \\
\hline & B & 1100 & 11,5 & Entre chave 008 e 009 & 35 \\
\hline & $\mathrm{C}$ & 350 & 5 & Entre chave 009 e 010 & 15 \\
\hline & D & 650 & 7,5 & Entre chave 010 e 011 & 23 \\
\hline & $E$ & 550 & 10 & Entre chave 011 e 006 & 30 \\
\hline
\end{tabular}

Fonte: Próprios Autores

Figura 6 - Informações gerais para simulação do impacto da interrupção no trecho

Abaixo, nas figuras 7 e 8, é possível analisar as simulações de DEC realizado em dois cenários, sendo:

i. Sem a utilização do sinalizador de falta; e 
ii. Com a utilização do sinalizador de falta, respectivamente.

\begin{tabular}{|c|c|c|c|c|c|c|}
\hline \multicolumn{7}{|c|}{$\begin{array}{l}\text { Simulação } 01 \text { - Desarme "circuito 1" sem sinalizador de falta com defeito entre a a chave } 004 \text { e } 005 \\
\text { Manobras, clientes afetados, tempo entre manobras e DEC entreas menobras de reenergização }\end{array}$} \\
\hline Manobra & A ou $F$ & Equipamento & $\begin{array}{l}\text { Qtd Clientes } \\
\text { Atingidos }\end{array}$ & $\begin{array}{c}\text { Clientes } \\
\text { Reenergizados }\end{array}$ & $\begin{array}{c}\text { Tempo entre manobras } \\
\text { (minutos) }\end{array}$ & Impacto DEC \\
\hline 1 & A & RD Circuito 1 & 3.200 & 0 & & \\
\hline 2 & A & 002 & 3.200 & 0 & 30 & \\
\hline 3 & $\mathrm{~F}$ & RD Circuito 1 & 2.350 & 850 & 0 & 0,004 \\
\hline 4 & A & 003 & 2.350 & 0 & 38 & \\
\hline 5 & $\mathrm{~F}$ & 002 & 1.700 & 650 & 38 & 0,006 \\
\hline 6 & A & 004 & 1.700 & 0 & 60 & \\
\hline 7 & $\mathrm{~F}$ & 003 & 1.200 & 500 & 23 & 0,005 \\
\hline 8 & A & 005 & 1.200 & 0 & 38 & \\
\hline 9 & $\mathrm{~F}$ & 004 & 450 & 750 & 15 & 0,002 \\
\hline 10 & A & RD Circuito 1 & 3.200 & 0 & 0 & \\
\hline \multicolumn{7}{|c|}{ Novo desarme devido ter sido alimentado o trecho do defeito } \\
\hline 11 & \begin{tabular}{l|l}
$A$ & \\
\end{tabular} & 004 & 3.200 & 0 & 0 & \\
\hline 12 & $\mathrm{~F}$ & RD Circuito 1 & 1.200 & 2.000 & 0 & \\
\hline 13 & $F$ & 006 & 750 & 450 & 30 & 0,000 \\
\hline \multicolumn{6}{|c|}{ Isolado trecho de defeito entre as chaves 004 e 005 ficando interrompidos 750 clientes até a localização do defeito } & 0,018 \\
\hline $\begin{array}{r}\text { Qte clie } \\
\text { Distribuidora } \mathrm{F}\end{array}$ & $\begin{array}{l}\text { tal da } \\
\text { culo de DEC }\end{array}$ & 455.000 & & & & \\
\hline
\end{tabular}

Fonte: Próprios Autores

Figura 7 - Simulação de DEC sem utilização de sinalizador de falta

Com base na figura acima é possível identificar o DEC em cada instante das manobras, bem como o impacto do DEC em seus respectivos momentos e os clientes atingidos/desenergizados. Compreende-se então, que para os dados analisados, houve um impacto de 0,018 no DEC da Distribuidora para a simulação 01.

Já na figura 8, abaixo, é possível analisar o mesmo caso em estudo, entretanto com a utilização dos sinalizadores de falta no trecho com defeito entre a chave 004 e 005, logo, observa-se uma redução de aproximadamente $25 \%$ de impacto DEC desta ocorrência técnica.

\begin{tabular}{|c|c|c|c|c|c|c|}
\hline \multicolumn{7}{|c|}{$\begin{array}{l}\text { Simulação } 02 \text { - Desarme "circuito 1" com sinalizador de falta com defeito entre a a chave } 004 \text { e } 005 \\
\text { Manobras, clientes afetados, tempo entre manobras e DEC entreas menobras de reenergização }\end{array}$} \\
\hline Manobra & $A$ ou $F$ & Equipamento & $\begin{array}{l}\text { Qtd Clientes } \\
\text { Atingidos }\end{array}$ & $\begin{array}{l}\text { Clientes } \\
\text { Reenergizados }\end{array}$ & $\begin{array}{c}\text { Tempo entre } \\
\text { manobras } \\
\text { (minutos) }\end{array}$ & Impacto DEC \\
\hline 1 & $\mathrm{~A}$ & RD Circuito 1 & 3200 & 0 & & \\
\hline 2 & $A$ & 003 & 3200 & 0 & 68 & \\
\hline 3 & $\mathrm{~F}$ & RD Circuito 1 & 1700 & 1500 & 0 & 0,008 \\
\hline 4 & A & 004 & 1700 & 0 & 23 & \\
\hline 5 & $\mathrm{~F}$ & 003 & 1200 & 500 & 23 & 0,003 \\
\hline 6 & A & 005 & 1200 & 0 & 38 & \\
\hline 7 & $\mathrm{~F}$ & 004 & 450 & 750 & 15 & 0,002 \\
\hline 8 & $\mathrm{~A}$ & RD Circuito 1 & 3200 & 0 & 0 & \\
\hline \multicolumn{7}{|c|}{ Novo desarme devido ter sido alimentado o trecho do defeito } \\
\hline 9 & A & 004 & 3200 & 0 & 0 & \\
\hline 10 & $\mathrm{~F}$ & RD Circuito 1 & 1200 & 2000 & 0 & \\
\hline 11 & $\mathrm{~F}$ & 006 & 750 & 450 & 30 & 0,000 \\
\hline \multicolumn{6}{|c|}{ Isolado trecho de defeito entre as chaves 004 e 005 ficando interrompidos 750 clientes até a localização do defeito } & 0,014 \\
\hline $\begin{array}{r}\text { Qte clientes } \mathbf{t} \\
\text { para c }\end{array}$ & $\begin{array}{l}\text { Distribuidora } \\
\text { DEC }\end{array}$ & 455.000 & & & Redução DEC $\Rightarrow$ & $-25 \%$ \\
\hline
\end{tabular}

Fonte: Próprios Autores

Figura 8 - Simulação de DEC com utilização de sinalizador de falta

Esta redução da duração equivalente da interrupção permite a concessionária aplicar seus esforços em outras demandas de cunho emergencial e/ou programado, fazendo com que a aplicação dos 
sensores de falta possa ser expandida cada vez mais como forma de minimizar os impactos à continuidade do fornecimento.

\section{CONCLUSÃO}

O estudo de caso da aplicação de sinalizadores de falta em rede de média tensão de uma distribuidora de energia elétrica do interior de Minas Gerais faz parte de um processo necessário de todas as Distribuidoras do país em identificar soluções práticas para reduzir a abrangência e duração das interrupções de energia elétrica, vez que, caso contrário, estão sujeitas à penalidades regulatórias por descumprimento de indicadores de continuidade, sejam elas pecuniárias ou até mesmo com a perca da concessão federal pelo órgão regulador, dependendo da criticidade e reincidências das ocorrências.

O sinalizador analisado, quando analisado em um caso hipotético sob mesmas circunstâncias, apresentou redução de $25 \%$ com sua aplicação, isto consiste em dizer que sua utilização proporciona às Distribuidoras mais um mecanismo de gestão de seus processos, oferecendo assim maior agilidade no reestabelecimento da energia elétrica, menores pagamentos com indenizações financeiras e, consequentemente, maior satisfação dos consumidores.

Conclui-se então que os sensores/sinalizadores de falta podem ser um grande aliado das concessionárias, trazendo agilidade no reestabelecimento de energia elétrica como uma de suas principais características. Logo, apesar de este não ser a única solução aplicada, tem-se utilizado a tecnologia cada vez mais como forma de ações mitigatórias, fazendo com que ações neste sentido sejam cada vez mais pertinentes no setor elétrico.

\section{REFERÊNCIAS}

AGÊNCIA NACIONAL DE ENERGIA ELÉTRICA - ANEEL. DGC - Desempenho Global de Continuidade (Ranking ANEEL-2019). Disponível em: https://www.aneel.gov.br/ranking-2019. Acesso em 18 de outubro de 2021.

AGÊNCIA NACIONAL DE ENERGIA ELÉTRICA - ANEEL. PRODIST Módulo 8. Disponível em: https://www.aneel.gov.br/modulo-8. Acesso em 15 de outubro de 2021.

AGÊNCIA NACIONAL DE ENERGIA ELÉTRICA - ANEEL. Serviço Público de Distribuição de Energia Elétrica. Disponível em: https://www.aneel.gov.br/distribuicao2 Acesso em 15 de outubro de 2021.

AGÊNCIA NACIONAL DE ENERGIA ELÉTRICA - ANEEL. PRORET - Submódulo 2.5A. Acesso em 13 de outubro de 2021.

AGÊNCIA NACIONAL DE ENERGIA ELÉTRICA - ANEEL Bem Vindo à ANEEL. Disponível em> https://www.aneel.gov.br/a-aneel. Acesso em 26/10/2021.

BERTULUCCI, Cristiano. Sensor: Você Sabe o Que é Quais os Tipos?. Disponível em: https://www.citisystems.com.br/sensor-voce-sabe-que-quais-tipos/. Acesso em 26/10/2021. 
MILANI, Álvaro Pereira; FARINAZZO, Bruno Flores; OLIVEIRA, Fábio Batista; CARDOSO, Mateus de Sousa; DE OLIVEIRA, Antônio Elízio.

Utilização de sensor de falta para localização de defeitos em redes de média tensão

IEEE-C37 Guide for Determining Fault Location on AC Transmission and Distribution Lines, IEEE Standard C37.114-2004, Jun. 2005

E. O. SCHWEITZER MANUFACTURING UMA DIVISÃO DA SEL. Sensores e Indicadores de Falta Catálogo 2008. 